\title{
Application in image-interpretation of the whole wave band CCD system

\author{
Wei Han ${ }^{a}$, Zhonglin $\mathrm{Xu}^{\mathrm{b}}$, Guobin Zhao ${ }^{\mathrm{c}}$,Nan Zheng ${ }^{\mathrm{d}}$,Jun Xie ${ }^{\mathrm{e}}$ \\ Aviation University of Air Force,Changchun,China, 130022 \\ ahwa3@163.com, ${ }^{b} x u z h o n g l i n @ s i n a . c o m,{ }^{c}$ sibindetianshi@sina.com , \\ dzhengnan@sina.com, ${ }^{\mathrm{e}} \mathrm{xiejun@163.com}$
}

\begin{abstract}
Keywords: Whole wave band CCD system; Image extraction;Image processing
\end{abstract}
\begin{abstract}
The whole wave band CCD system is widely used for examining purple outside, visible light, infrared picture high-resolution show and professional pattern process equipment in real time. It can visible light picture turn visible light picture into in real time, can realize faint collection and high-quality collection and processing of picture of signal under the low intensity of illumination situation, incorporate the verification of traces, file are examined, figure taking pictures and pattern process into an organic whole.
\end{abstract}

\section{Introduction}

The whole wave band CCD camera is developed at first by U.S.A., apply to study the technological field in spaceflight, army industry science at first, this equipment adopts and lengthens time for exposure and method to reduce noise mainly, in a situation that the intensity of illumination is low or the mark material evidence is very faint, can get the comparatively clear picture, at the same time, can comparatively lower the interference of the background of the object effectively too, it is one of the most advanced taking pictures tools at present. The whole wave band CCD system shows and professional pattern process equipment in real time purple outside of one set, visible light, infrared picture high-resolution. It can visible light picture turn visible light picture into in real time, can realize faint collection and high-quality collection and processing of picture of signal under the low intensity of illumination situation, incorporate the verification of traces , file are examined, figure taking pictures and pattern process into an organic whole.

The whole system , CCD of wave band, adopt depth half conductor refrigeration technology when design, when the external environment temperature is 20 degrees Centigrade, the minimum refrigeration temperature can be up to- 70 degrees Centigrade, can reduce the dark current through reducing working temperature, improve SNR, realize that is exposed for a long time, catch the extremely faint signal. The system adopted the total mark to expose technology, at the same time quantization got strong signal and weak signal in the same picture . Its sensitization range 160nm$1100 \mathrm{~nm}$, it was black-and-white picture that was written down, it is the color reflecting and writing down the picture with the gray steps that is left with the level, its grey level grade is from 1 to 65553, choose systematic gaining and reading the speed through the software while shooting, can obtain the mark material evidence picture that contrast strengthened, to some mark pictures with extremely faint contrast, its characteristic of display that it can be very good. The sensitive wave band of the whole wave band CCD system is wide, the sensitive range of spectrum of this system is $200 \mathrm{~nm}-1200 \mathrm{~nm}$, can carry on purple other district, visible light district, infrared region and take pictures etc.

\section{Images drawing and analysing}

Introduction to the experiment platform

The size or picture of laser scanning based on CCD system shows measures the experimental provision. Three-dimensional laser scanning that device adopt this, overcome traditional vision machine succeed the two-dimentional picture three-dimensional scenery projection, cause and construct the three-dimensional scenery only defect again. Survey and draw and gather clicking 
more under this system (at least several ten thousand, more than several million at most), the depth of field of the picture is long, make the treated data true and reliable.

The structure of the experimental provision and size of laser scanning of operation principle measure the device system and adopt the single track mere law of structure,this system including semiconductor laser instrument, CCD camera , picture gather card, computer, measuring platform , measuring and pattern process software etc..

The systematic flow chart is as Fig. 1 shows. The system regards semiconductor laser instrument as the light source, its light beam is become on the fan-shaped level of the vertical direction only shone and examined the thing by the optical system, being examined and forming the stripe on the thing. Sloping and observing this stripe to get a stripe picture with CCD camera, the data information of the income is gathered the card by the picture and sent into the computer and dealt with from the software of measuring, according to geometrical optics and this stripe deformation along $\mathrm{z}$ high change, axle of direction conversion of pattern triangular law. And is examined the thing and moved according to certain law in certain direction, so can receive the depth picture information on the object surface .

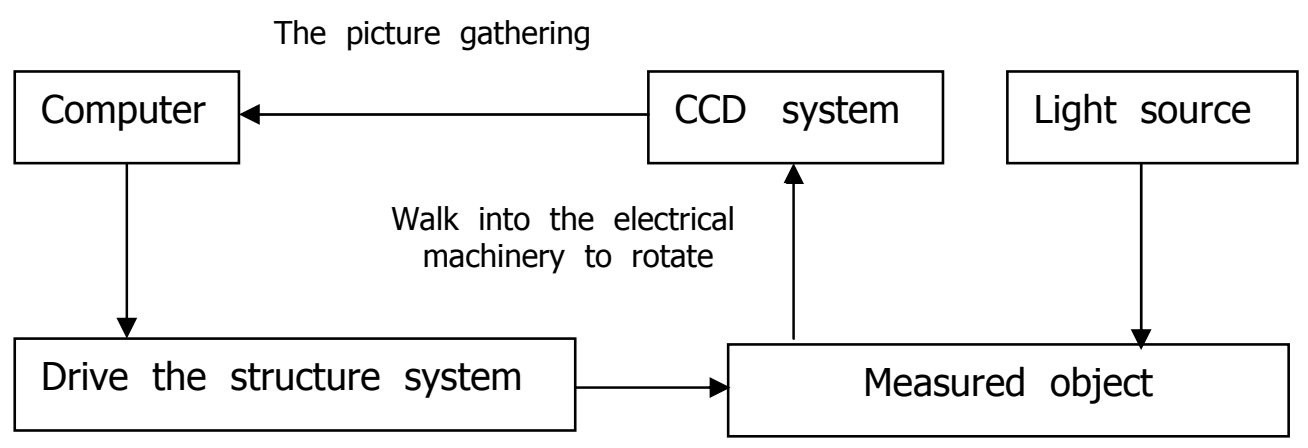

Fig 1. Systematic job flow chart

Measure the material object and adopt diameter $5 \mathrm{~mm}$, the cylindrical part of high $80 \mathrm{~mm}$, walking into amount $2 \mathrm{~mm}$, the system adopts the standard volume one standardly, a distance clicked with consult coordinates of measuring amount is as the standard depth, measure a height and is regarded as the standard height. Can know from the result of measuring, the data measured move towards the form with the clause to accord with, can guarantee the precision. The threedimensional laser scanning that the size of laser scanning based on CCD measures the experimental provision and adopts is different from simple survey and drawing technology, it can describe the intact attribute of the goal structure, is suitable for the original appearance of reducing the more goal.

\section{Application of the whole wave band CCD system in image technology}

\section{Inspection for the ammunition leftovers}

Launch ammunition after remain mark have barium salt, appear orange fluorescence particle form distribute until the whole system , CCD of wave band, observe, but as differentiating the basis of launching the entry, the experiment indicates, adopt the whole wave band CCD system to shoot apter existence of observing the barium salt particle in the room on the black carrier.

2. For fighting the inspection of place shape or ground form

The modern battle field is by enormous C4I system that light, electronics and information network disposing on the multidimensional space make up, among them the information subsystem is one of the main backbones. Through adopting various kinds of and detecting and examining the characteristic information that technology and means obtain enemy's goal in one's own side's army, rely on the medium (the air, sea water, earth ) to convey to the acception station directly or in the form of sound, light, electric wave, processing, showing writing down, interpretation, the course 
of analysing etc. is turned into the available information. The modern battle field situation is fast changing, the commander will not establish in an unassailable position unless grasp enemy and our accurate situations of both sides in time. So the intelligence work must be continuous, real-time, information processing automation. No matter take any means, it is an essential procedure to detect and examine. According to the characteristics of the object and electromagnetic wave, scientific and technical personnel develop the varied reconnaissance means, for example: The visible light reconnoiters the technology and equipment, the shimmer reconnoiters the technology and equipment, reconnoiter the technology and equipment infraredly, reconnoiter the equipment and reconnoiter the technology and equipment, the sensor reconnoiters the technology and equipment, the satellite reconnoiters etc.. It is to utilize the electromagnetic wave launched to shine the goal and receive its return waves echo that these reconnoiter the means, therefore come to find the goal and locating, movement direction and the speed and other characteristics. Regard reconnoitering the aerial electric wave of the equipment and reflecting the centre as the radial coordinates origin, survey the distance, azimuth and angle of pitch of goals, some can survey goal speed, picture also, in addition can also through airborne CCD as move system base station is it measure the method while being above-mentioned to finish.

3. Inspection for the fingerprint, footprint or body fluid

In the course of using the whole wave band CCD system and carrying on fingerprint to examine, can adopt and examine the method that technology is similar to with laser fingerprint , including examine the law, dyestuffs and cover membrane powder law, three ketone of indene directly / chlorine zinc law, dyestuffs float and dye the law, dyestuffs solution to float and dye the law etc., observation of the material evidence of suitable for potential fingerprint, staining the fingerprint, bloodstain, footprint with blooding etc., drawing and examining. The potential fingerprint on the plastic products after the cyanogen acrylic acid fat is dealt with, and then float and dye with Rodin bright 6G dyestuffs, observe under the indoor light and the whole wave band CCD system separately, the latter fluorescence is strong, fingerprint appears clearly. Three ketone derivatives of indene developed in recent years, cooperate with the chlorine treated fingerprint of zinc solution, it will produce a lot of fluorescence results too that the whole wave band CCD system is observed, it is with high efficiency that the fluorescence gives out light, traditional three ketone laws of indene that superior to. Equally, there is strong fluorescence too under the whole wave band CCD system to examine chemical medicines DFO used in technology in laser fingerprint. In a word, use the whole wave band CCD system to dispel the mark background to interfere effectively, strengthen image contrast.

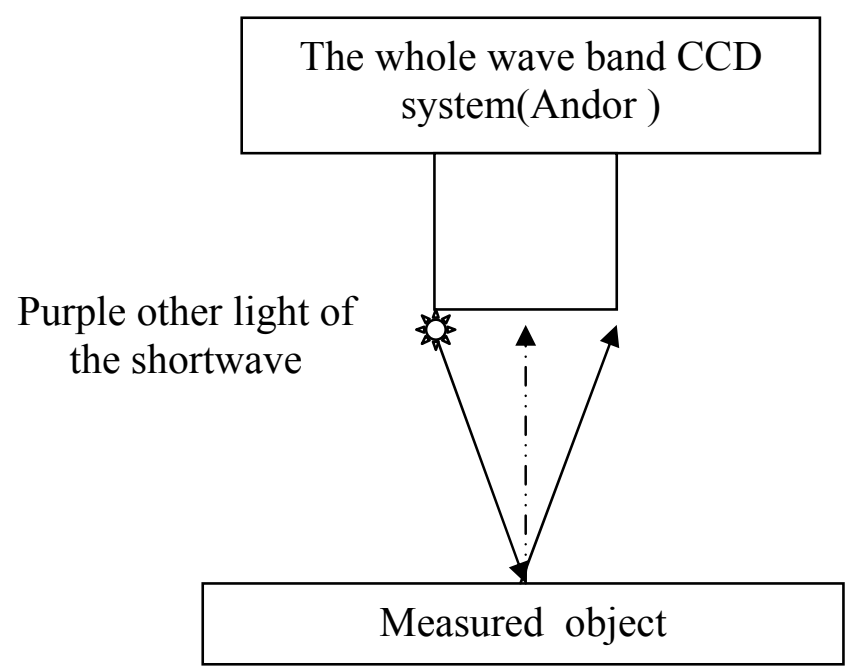

Fig 2. Check under the shortwave ultraviolet ray

in the whole wave band CCD system

CCD system can be used for appearing to the footprint in addition. The footprint adopted at 
present has physics method, chemical method and optics method, etc. to appear . In the optics method: to mix and only take pictures the method, colour separation and take pictures the method, polarized light to take pictures the method and purple outside and reflect the method of taking pictures. To the optics method, can all shoot appearing with the whole wave band CCD system. As to ordinary camera, the whole wave band CCD system is in the method of appearing of optics, it is quicker that it manifests the speed, and it is even more ideal to manifest the result, especially it is more obvious to reflect its advantage while taking pictures purple outside. For example to the dust footprint on some objects with comparatively complicated background, dispel the interference of the background pattern, generally need to reflect the method of taking pictures to appear with purple outside, and need to plunder mixing all angle of the incidence. But because the footprint is relatively large in area, if shoot and manifest taking the time for exposure of 15-30 minutes the same with the ordinary camera. And easy to expose appearing evenly. If over-exposed close to the purple other light position, but it is not enough to far away from purple other light position and expose, have influenced the appearing of footprint detail position. Can overcome this defect effectively with the whole wave band CCD system. Time for exposure when it needed is 1-5 minutes, can adjust each position of whole footprint to expose evenly at the same time . Enable outputting the picture and keep unanimity on contrast and bright degree. In addition, it produces the fluorescence under the circumstances that the whole wave band CCD system is observed that the human body, such as seminal fluid, serum, saliva, oil, etc. secrete, therefore can discern its spot mark position easily, especially it is more convenient to examine the body fluid on the dark clothes.

\section{Discussion}

With the development of science and technology, the digitized picture of direct output of the whole wave band CCD system, can also be real-time check-up is revised, the digitized process of making the whole information reconnoiter is accelerated greatly. The appearance of this system, the network enabling interpretation personnel and pattern process personnel to totally realize the digitized picture is real-time and mutual. Take a picture while manifesting various kinds of topographical ground form or the mark material evidence with the whole wave band CCD system, besides mixing the light and focusing, other work is finished in the computer, and the picture got is all right appear " real-timly " on the monitor. Especially to take pictures to purple outside and infraredly, all right to be " real-time " sees the picture, so can adjust and match all angle according to the power of contrast, avoid " striking blindly ". The picture that meanwhile takes a picture and writes down can revise check-up in the computer, then can type out directly, develop and print the link of putting etc. after reducing the sensitization film, have improve working efficiency greatly, has reconnoitered the real-time character, validity obtained and established the solid foundation for the information. But the one that need to pay attention to at the same time is, as the conditions , such as light source, illumination, etc. are limited and influenced, what a deep question that thinks that the effective one goes to make an uproar to become worth.

\section{References}

[1] Xu XiaoJing, Lin Jing. The material evidence of the whole wave band examines application [J] in the field of criminal camera work of CCD system. Image technology, 2003 (3).

[2] Cao Ri gets up. Technical research of distance finding of laser scanning [D] on the basis of CCD. Harbin: Harbin Engineer University, 2006.

[3] Qiu Ying, Tan DingZhong, etc.. Measure the reseach [J] of the experimental provision on the basis of CCD laser scanning measurement. Laboratory research and exploring, 2011 (3).

[4] Zhang ShengYun, Zhang LiJiang. The whole wave band CCD reflects the application [J] while taking pictures purple outside. Criminal technique, 2008 (1).

[5] Chen EnTao, Lu E. Hot design of CCD package [J] of space remote sensing device. The precision engineering of optics, 2000 (8).

[6] Sun ChangKu, Ye ShengHua. The laser measures the technology [M]. Tianjin: The publishing 
house of university of Tianjin, 2001.

[7] Old banyan front yard, Peng MeiGui. CCD/CMOS picture sensor foundation and using [M]. Beijing: Science Press, 2006.

[8] Nie XiaoXiao. Application [J] of the whole wave band CCD system in criminal image technology. Legal system and society, 2011 (2). 